\title{
Modulational Instability and Generation of Envelope Solitons in Four Component Space Plasmas
}

\author{
N. A. Chowdhury, A. Mannan, M. R. Hossen, and A. A. Mamun
}

\begin{abstract}
A four component space plasma system (consisting of immobile positive ions, inertial cold positrons as well as hot electrons and positrons following Cairns' nonthermal distribution function is considered. The nonlinear propagation of the positronacoustic (PA) waves, in which the inertia (restoring force) is provided by the cold positron species (nonthermal pressure of both hot electron and positron species) has been theoretically investigated by deriving the nonlinear Schrödinger (NLS) equation. It is found from the numerical analysis of this NLS equation that the space plasma system under consideration supports the existence of both dark and bright envelope solitons associated with PA waves, and that the dark (bright) envelope solitons are modulationally stable (unstable). It is also observed that the basic properties (viz. stable regime and unstable regime with growth rate) of the PA envelope solitions are significantly modified by related plasma parameters (viz. number densities and temperature of plasma species), which correspond to different realistic space plasma situations.
\end{abstract}

Index Terms-Positron-acoustic waves, Modulational instability, Envelope solitons.

\section{INTRODUCTION}

Nowadays, the physicists are mesmerized by the natural beauty of electron-positron-ion (e-p-i) plasmas because many painstaking observations disclosed the existence of e-p-i plasmas in various regions of our universe (such as supernovas, pulsar environments, cluster explosions [1]-[3], etc.), polar regions of neutron stars [4], white dwarfs [5], [6], early universe [7], inner regions of the accretion disc surrounding black holes [8], pulsar magnetosphere [9], [10], center of our galaxy [11], and solar atmospheres [12], [13].

To understand the physics of collective processes in such kind of plasmas, many researchers have studied the ionacoustic waves (IAWs) [14]-[16] and electron-acoustic waves (EAWs) [17], [18] in e-p-i plasmas. A few of them have considered isothermal Maxwellian distribution [19]-[23], for their considered plasma species. But in astrophysical environments generally a nonthermal plasma (present of excess nonMaxwellian particles such as electrons and positrons, which is absent from thermodynamic equilibrium, that means at least one of the component of such kind of plasmas does not follow the prominent Maxwell-Boltzmann distribution) are characterized by long tail in the high- energy region [24], around the Earth's bow shock [25], lower part of magetosphere [26], and upper Martin ionosphere [27]. By employing nonthermal

N. A. Chowdhury, A. Mannan, and A. A. Mamun are with the Department of Physics, Jahangirnagar University, Savar, Dhaka, Bangladesh.

M. R. Hossen is with the Department of General Educational Development, Daffodil International University, Dhanmondi, Dhaka-1207, Bangladesh.

Corresponding author's e-mail: nurealam1743phy@gmail.com distribution for plasma species instead Maxwellian distribution in the highly populated nonthermal particles region, obtained result is comparatively more acceptable with, which observed by Freja and Viking Satellites [26], [28]. So for better understanding about this high energetic space plasmas nonthemal distribution can be used to model such kind of space plasma system.

A set of researchers have been used nonthermal distribution to study linear and nonlinear structure of e-p-i plasmas. Like Cairns et al. [29] used nonthermal distribution of electrons to understand, how the presence of a population of energetic electrons changes the nature of ion sound solitary waves. By using pseudo-potential method, Pakzad [31] studied that under certain criteria, the formation of solitons and the effect of nonthermal electrons on solitons in e-p-i plasmas. Sahu [32] analyzed the effects of ion kinematics viscosity on the properties of PA Shock Waves. Messekher et al. [33] examined the influence of quantum effects on solitary (quantum positronacoustic waves) structures as well as double-layers by deriving Korteweg-de Vries equation in an unmagnetized four component plasmas. By employing the reductive perturbation method (RPM), Eslami et al. [34] investigated modulational instability (MI) of IAWs in q-nonextensive e-p-i plasmas. Sultana and Kaurakis [17], by making use of a multiscale perturbation technique, a NLS equation is derived to examined the stability of the EAWs and formation of envelope solitons under certain conditions in e-i plasmas. Zhang et al. [35] studied the MI for e-p-i plasma system and observed that the amplitude of dark and bright envelope solitons significantly depends on the effects of nonthermal parameter, concentration of positrons and ion temperatures. Up to the best of our knowledge, no theoretical investigations have been worked out about the nonlinear properties of positron-acoustic waves (PAWs) in unmagnetized plasmas with immobile ions, inertial cold positrons, nonthermal distributed hot electrons and positrons. Therefore, in our present work, we attempt to study the MI of PAWs and formation of envelope solitons by deriving the NLS equation in a plasma having excess of nonthermal distributed hot electrons and positrons in a "non-Maxwellian tail".

The present paper is organized as follows. The basic governing equations of our plasma model are presented in Sec. II. By using perturbation technique, we derive a NLS equation which governs the slow amplitude evolution in time and space in Sec. III. The stability analysis is presented in Sec. IV. Envelope solitons are devoted in Sec. V. Conclusion is preserved in sec.VI. 


\section{GOVERNING EQUATIONS}

We consider an unmagnetized four component plasma system consisting of immobile positive ions, inertial cold positrons, nonthermally distributed hot electrons and hot positrons. At equilibrium, the quasi-neutrality condition can be expressed as $n_{c p 0}+n_{h p 0}+n_{i 0}=n_{e 0}$, where $n_{c p 0}, n_{h p 0}, n_{i 0}$, and $n_{e 0}$ are the unperturbed number densities of cold positron, hot positron, immobile ion, and hot electron respectively. The normalized governing equations of the PAWs in our considered plasma system are given by

$$
\begin{aligned}
& \frac{\partial n_{c p}}{\partial t}+\frac{\partial}{\partial x}\left(n_{c p} u_{c p}\right)=0, \\
& \frac{\partial u_{c p}}{\partial t}+u_{c p} \frac{\partial u_{c p}}{\partial x}=-\frac{\partial \phi}{\partial x}, \\
& \frac{\partial^{2} \phi}{\partial x^{2}}=-n_{c p}-\mu_{1} n_{h p}+\mu_{2} n_{e}-\mu_{3} .
\end{aligned}
$$

For inertialess hot positrons and hot electrons are given by the following expression,

$$
\begin{aligned}
& n_{h p}=\left(1+\beta \sigma_{1} \phi+\beta \sigma_{1}^{2} \phi^{2}\right) \exp \left(-\sigma_{1} \phi\right), \\
& n_{e}=\left(1-\beta \sigma_{2} \phi+\beta \sigma_{2}^{2} \phi^{2}\right) \exp \left(\sigma_{2} \phi\right) .
\end{aligned}
$$

Substituting equation (4) into equation (3), and expanding up to third order, we get

$$
\begin{aligned}
\frac{\partial^{2} \phi}{\partial x^{2}}=-n_{c p}-\mu_{1}+\mu_{2} & -\mu_{3}+\gamma_{1} \phi+\gamma_{2} \phi^{2} \\
& +\gamma_{3} \phi^{3}+\cdots \cdots \cdots
\end{aligned}
$$

where

$$
\begin{aligned}
& \gamma_{1}=(1-\beta)\left(\mu_{1} \sigma_{1}+\mu_{2} \sigma_{2}\right), \\
& \gamma_{2}=\left(\mu_{2}^{2} \sigma_{2}^{2}-\mu_{1}^{2} \sigma_{1}^{2}\right) / 2, \\
& \gamma_{3}=(1+3 \beta)\left(\mu_{1} \sigma_{1}^{3}+\mu_{2} \sigma_{2}^{3}\right) / 6,
\end{aligned}
$$

and $\quad \sigma_{1}=\frac{T_{e f f}}{T_{h p}}, \quad \sigma_{2}=\frac{T_{e f f}}{T_{e}}, \quad \mu_{1}=\frac{n_{h p 0}}{n_{c p 0}}$,

$$
\mu_{2}=\frac{n_{e 0}}{n_{c p 0}}, \quad \mu_{3}=\frac{n_{i 0}}{n_{c p 0}}, \quad T_{e f f}=\frac{T_{e} T_{h p}}{\mu_{1} T_{e}+\mu_{2} T_{h p}} .
$$

In the above equations, the cold positron number density $n_{c p}$ is normalized by its unperturbed number density $n_{c p 0}$; $u_{c p}$ is the cold positron fluid speed normalized by the PA wave speed $C_{c p}=\left(k_{B} T_{e f f} / m_{p}\right)^{1 / 2} ; \phi$ is the electrostatic wave potential normalized by $k_{B} T_{e f f} / e$; where $k_{B}$ being the Boltzmann constant, $T_{\text {eff }}$ being the effective temperature, $m_{p}$ being the positron rest mass, and $e$ being the magnitude of single electron charge. The time and space variables are normalized by $\omega_{c p}^{-1}=\left(m_{p} / 4 \pi e^{2} n_{c p 0}\right)^{1 / 2}$ and $\lambda_{D p}=\left(k_{B} T_{e f f} / 4 \pi e^{2} n_{c p 0}\right)^{1 / 2}$ respectively.

\section{DERIVATION OF THE NLS EQUATION}

To study the modulation of the PAWs in our considered plasma system, we will derive the NLS equation by employing the reductive perturbation method. So we first introduce the independent variables are stretched as

$$
\xi=\epsilon\left(x-v_{g} t\right), \quad \tau=\epsilon^{2} t,
$$

where $v_{g}$ is the envelope group velocity to be determined later and $\epsilon(0<\epsilon<1)$ is a small (real) parameter. Then we can write a general expression for the dependent variables as

$$
\begin{aligned}
& M(x, t)=M_{0}+\sum_{m=1}^{\infty} \epsilon^{(m)} \sum_{l=-\infty}^{\infty} M_{l}^{(m)}(\xi, \tau) \exp (i l \Theta) \\
& M_{l}^{(m)}=\left[n_{p c l}^{(m)}, u_{p c l}^{(m)}, \phi_{l}^{(m)}\right]^{T}, \quad M_{l}^{(0)}=[1,0,0]^{T}
\end{aligned}
$$

where $\Theta=k x-\omega t$, where $\mathrm{k}$ and $\omega$ are real variables representing the carrier wave number and frequency, respectively. $M_{l}^{(m)}$ satisfies the pragmatic condition $M_{l}^{(m)}=M_{-l}^{(m)^{*}}$, where the asterisk denotes the complex conjugate. The derivative operators in the above equations are treated as follows:

$$
\frac{\partial}{\partial t} \rightarrow \frac{\partial}{\partial t}-\epsilon v_{g} \frac{\partial}{\partial \xi}+\epsilon^{2} \frac{\partial}{\partial \tau}, \quad \frac{\partial}{\partial x} \rightarrow \frac{\partial}{\partial x}+\epsilon \frac{\partial}{\partial \xi}
$$

Substituting equations $(6)-(8)$ into equations $(1),(2)$, and (5) and collecting the power terms of $\epsilon$, the first order $(m=1)$ equation with $(l=1)$ give

$$
\begin{aligned}
& -i \omega n_{1}^{(1)}+i k u_{1}^{(1)}=0, \quad-i \omega u_{1}^{(1)}+i k \phi_{1}^{(1)}=0, \\
& n_{1}^{(1)}-k^{2} \phi_{1}^{(1)}-\gamma_{1} \phi_{1}^{(1)}=0 .
\end{aligned}
$$

The solution for the first harmonics read as

$$
n_{1}^{(1)}=\frac{k^{2}}{\omega^{2}} \phi_{1}^{(1)}, \quad u_{1}^{(1)}=\frac{k}{\omega} \phi_{1}^{(1)} .
$$

We thus obtain the dispersion relation for PAWs

$$
\omega^{2}=\frac{k^{2}}{\left(k^{2}+\gamma_{1}\right)}
$$

The second-order when $(m=2)$ reduced equations with $(l=$ 1) are

$$
\begin{aligned}
& n_{1}^{(2)}=\frac{k^{2}}{\omega^{2}} \phi_{1}^{(2)}+\frac{2 i k\left(v_{g} k-\omega\right)}{\omega^{3}} \frac{\partial \phi_{1}^{(1)}}{\partial \xi}, \\
& u_{1}^{(2)}=\frac{k}{\omega} \phi_{1}^{(2)}+\frac{i\left(v_{g} k-\omega\right)}{\omega^{2}} \frac{\partial \phi_{1}^{(1)}}{\partial \xi}
\end{aligned}
$$

with the compatibility condition

$$
v_{g}=\frac{\partial \omega}{\partial k}=\frac{\omega\left(1-\omega^{2}\right)}{k} .
$$

The amplitude of the second-order harmonics are found to be proportional to $\left|\phi_{1}^{(1)}\right|^{2}$

$$
\begin{array}{ll}
n_{2}^{(2)}=C_{1}\left|\phi_{1}^{(1)}\right|^{2}, & n_{0}^{(2)}=C_{4}\left|\phi_{1}^{(1)}\right|^{2}, \\
u_{2}^{(2)}=C_{2}\left|\phi_{1}^{(1)}\right|^{2}, & u_{0}^{(2)}=C_{5}\left|\phi_{1}^{(1)}\right|^{2}, \\
\phi_{2}^{(2)}=C_{3}\left|\phi_{1}^{(1)}\right|^{2}, & \phi_{0}^{(2)}=C_{6}\left|\phi_{1}^{(1)}\right|^{2},
\end{array}
$$


(a)

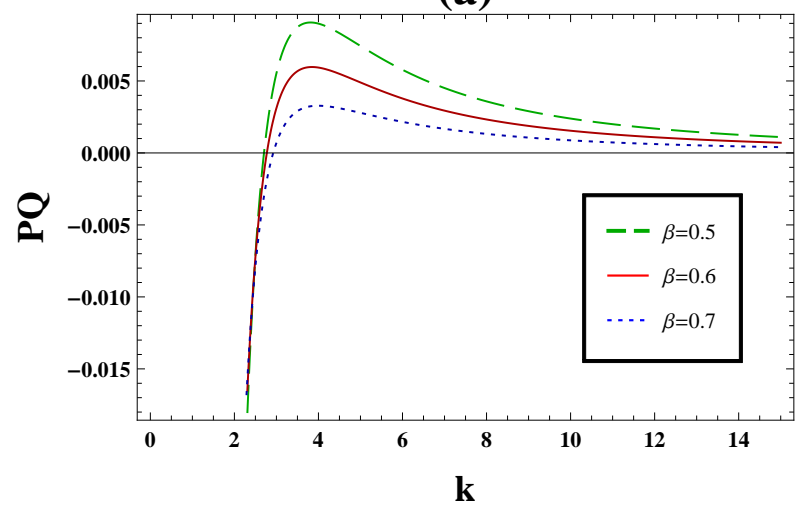

(b)

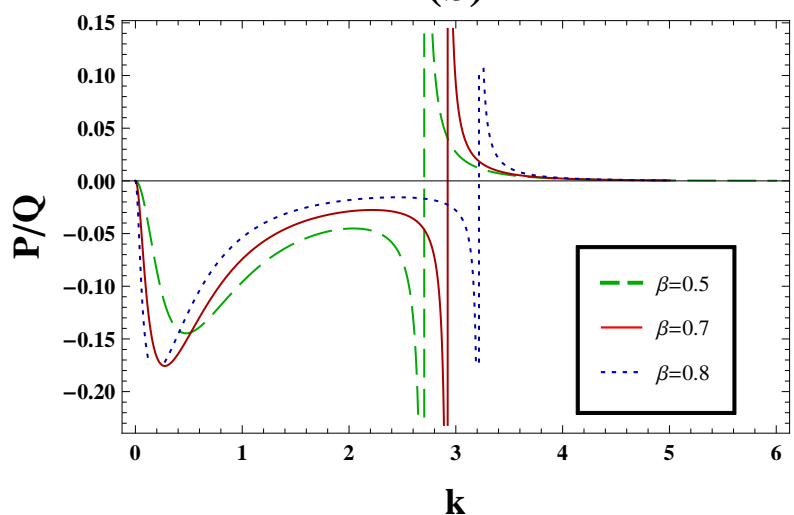

Fig. 1. (Color online)The Variation of $P Q$ or $P / Q$ with wave number $k$ for different values of $\beta$. (a) $P Q$ against $k$ for $\beta$, (b) $P / Q$ against $k$ for $\beta$. All the figures are generated by using these values, $\mu_{1}=0.2, \mu_{2}=0.7, \sigma_{1}=3, \sigma_{2}=1.5$ and $\beta=0.5$.

where

$$
\begin{aligned}
& C_{1}=\frac{3 k^{4}}{2 \omega^{4}}+\frac{C_{3} k^{2}}{\omega^{2}}, \\
& C_{2}=\frac{k^{3}}{2 \omega^{3}}+\frac{C_{3} k}{\omega}, \\
& C_{3}=\frac{3 k^{4}-2 \gamma_{2} \omega^{4}}{2 \omega^{4}\left(4 k^{2}+\gamma_{1}\right)-2 \omega^{2} k^{2}} . \\
& C_{4}=\frac{2 v_{g} k^{3}+\omega k^{2}+C_{6} \omega^{3}}{v_{g}^{2} \omega^{3}}, \\
& C_{5}=\frac{k^{2}+C_{6} \omega^{2}}{v_{g} \omega^{2}}, \\
& C_{6}=\frac{2 v_{g} k^{3}+\omega k^{2}-2 \gamma_{2} v_{g}^{2} \omega^{3}}{\gamma_{1} v_{g}^{2} \omega^{3}-\omega^{3}} .
\end{aligned}
$$

Finally, the third harmonic modes $(m=3)$ and $(l=1)$ and with the help of equations $(10)-(14)$, give a system of equations, which can be reduced to the following NLS equation:

$$
i \frac{\partial \Phi}{\partial \tau}+P \frac{\partial^{2} \Phi}{\partial \xi^{2}}+Q|\Phi|^{2} \Phi=0,
$$

where $\Phi=\phi_{1}^{(1)}$ for simplicity. The dispersion coefficient $P$ is

$$
P=\frac{1}{2} \frac{\partial v_{g}}{\partial k}=-\frac{3}{2} \frac{\omega^{2}}{k} v_{g},
$$

and the nonlinear coefficient $\mathrm{Q}$ is

$$
\begin{array}{r}
Q=\frac{\omega^{3}}{2 k^{2}}\left[-\frac{k^{2}\left(C_{1}+C_{4}\right)}{\omega^{2}}+2 \gamma_{2}\left(C_{3}+C_{6}\right)\right. \\
\left.+3 \gamma_{3}-\frac{2 k^{3}\left(C_{2}+C_{5}\right)}{\omega^{3}}\right] .
\end{array}
$$

\section{Stability ANALYSIS}

The evolution of PAWs are governed by the equation (15) essentially depends on the coefficients product $P Q$. Let us consider the harmonic modulated amplitude solution $\Phi=\Phi_{o} \exp \left(i Q\left|\Phi_{o}\right|^{2} \tau\right)$. Following the standard stability analysis, one may perturb the amplitude by setting $\Phi=$ $\hat{\Phi}_{0}+\epsilon \hat{\Phi}_{1,0} \exp \left[i\left(k_{M I} \xi-\omega_{M I} \tau\right)\right]+c . c$ (the perturbation wave number $k_{M I}$ and the frequency $\omega_{M I}$ ). Hence, the nonlinear dispersion relation for the amplitude modulation [36], [37] is given by

$$
\omega_{M I}^{2}=P^{2} k_{M I}^{2}\left(k_{M I}^{2}-2 \frac{Q}{P}\left|\Phi_{o}\right|^{2}\right) .
$$

Clearly, if $P Q<0, \omega_{M I}$ is always real for all values of $k_{M I}$, hence in this region the PAWs are stable in the presence of small perturbation. On the other hand, when $P Q>0$, the MI would set in as $\omega_{M I}$ becomes imaginary and the PAWs are unstable for $k_{M I}<k_{c}=\sqrt{2 Q\left|\Phi_{o}\right|^{2} / P}$, where $k_{c}$ is the critical value of the wave number of modulation and $\Phi_{o}$ is the amplitude of the carrier waves. The growth rate $\left(\Gamma_{g}\right)$ of MI (within this conditions, when $P Q>0$ and simultaneously $\left.k_{M I}<k_{c}\right)$ is given by

$$
\Gamma_{g}=|P| k_{M I}^{2} \sqrt{\frac{k_{c}^{2}}{k_{M I}^{2}}-1} .
$$

Clearly, the maximum value $\Gamma_{g(\max )}$ of $\Gamma_{g}$ is obtained at $k_{M I}=k_{c} / \sqrt{2}$ and is given by $\Gamma_{g(\max )}=|Q|\left|\Phi_{0}\right|^{2}$.

The coefficients of dispersion term $P$ and nonlinear term $Q$ are dependent on various physical plasma parameters, such as $\sigma_{1}, \sigma_{2}, \mu_{1}, \mu_{2}$, and $\beta$. Thus, these parameters may be sensitive to change the stability conditions of the PAWs. One can recognize the stability conditions of PAWs by depicting $P / Q$ against $k$ for different physical plasma parameters. The stability of the profile is depicted in Figs. $1(b)$ and 2, where it is shown that the variation of the ratio of $P / Q$ versus $k$ for different plasma parameters. When the sign of the ratio $P / Q$ is negative, the PAWs are modulationally stable, while the sign of the ratio $P / Q$ is positive, the PAWs will be modulationally unstable against external perturbations. It is clear that both stable and unstable region for PAWs are obtained from the Figs. $1(s)$ and 2 . When $P / Q \rightarrow \pm \infty$, the corresponding value of $k\left(=k_{c}\right)$ is called critical or threshold wave number for the onset of MI. This critical value separates the unstable $(P / Q>$ $0)$ from the stable region $(P / Q<0)$ one.

The nonthermal parameter plays a significant role to change the stability of the PAWs. With the increasing values of $\beta$ (nonthermality), the critical value $k_{c}$ is shifted to the higher 
(a)

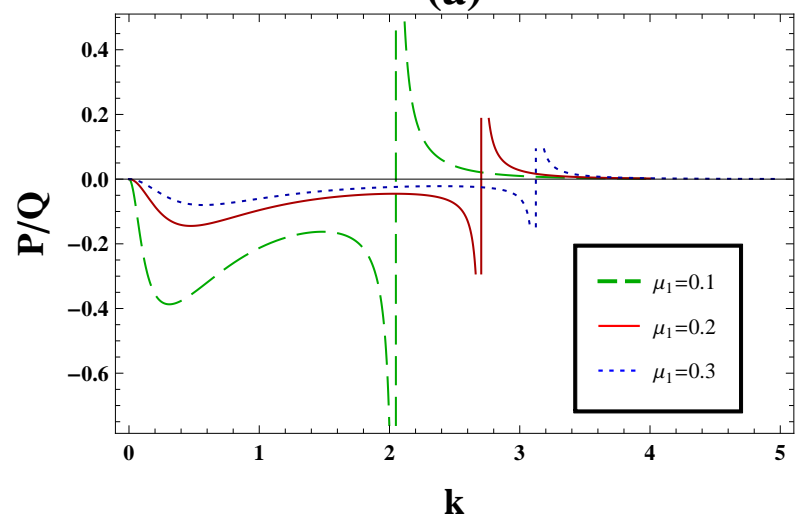

(c)

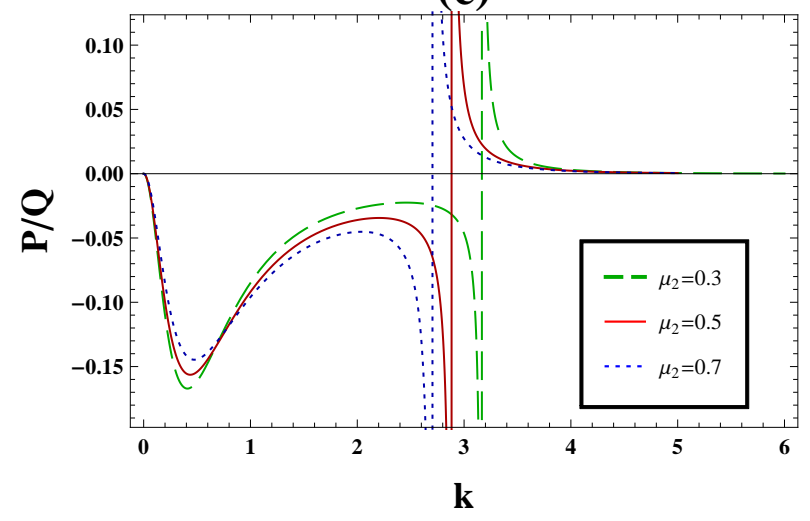

(b)

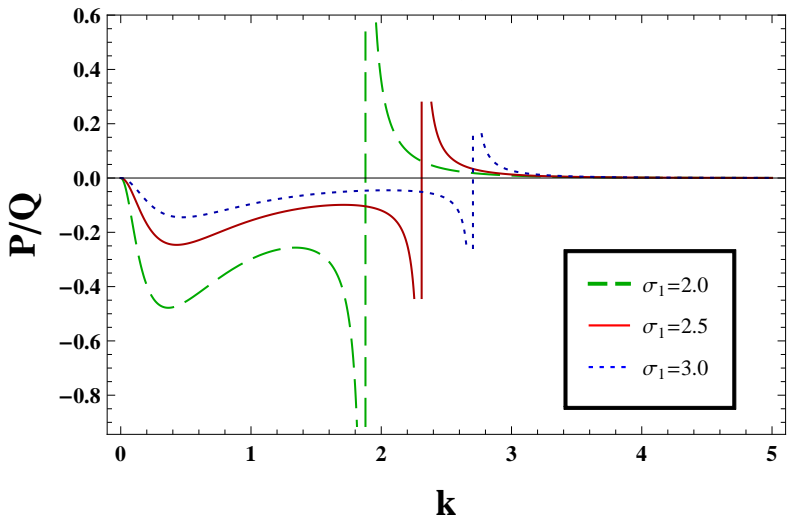

(d)

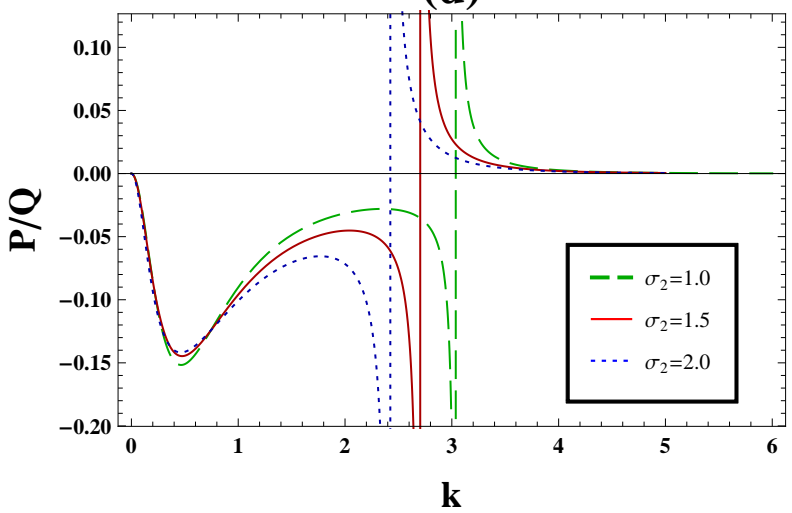

Fig. 2. (Color online) The Variation of $P / Q$ against $k$ for different values of plasma parameters. (a) For $\mu_{1}$, (b) For $\sigma_{1}$, (c) For $\mu_{2}$ and (d) For $\sigma_{2}$.

(a)

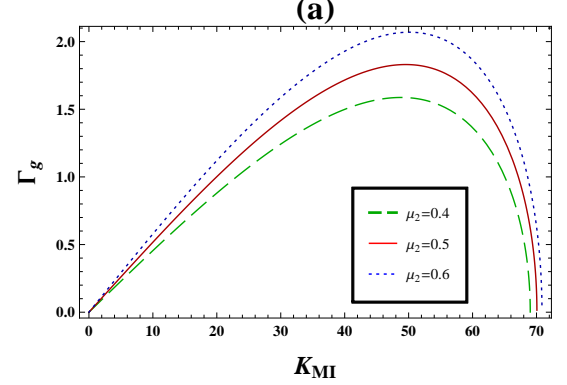

(b)

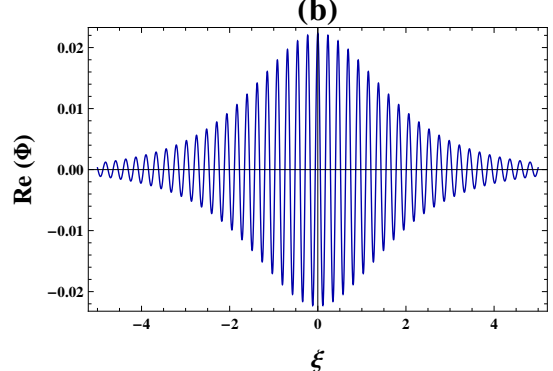

(c)

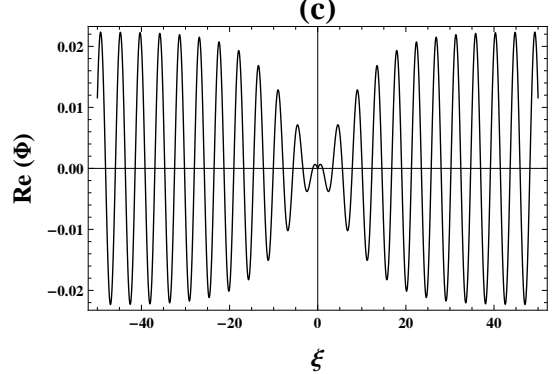

Fig. 3. (Color online) Plot of the MI growth rate $\left(\Gamma_{g}\right)$ against $k_{M I}$ for different values of $\mu_{2}$, along with $k=6$, and $\Phi_{0}=0.6$. (b) Bright envelope solitons for $k=5$, (c) Dark envelope solitons for $k=2.2$. Along with $\psi_{0}=0.0005, U=0.1, \tau=0$, and $\Omega_{0}=0.4$.

value (see Fig. 1(b)). It is seen that the instability sets increases with the increasing values of nonthermal parameter $\beta$. It is also found that the absolute value of the ratio $P / Q$ increases with the increasing values of $\beta$.

Figure 2(a) shows the variation of $P / Q$ with $k$ for different values of hot to cold positron concentration ratio (via $\mu_{1}$ ) with fixed values of other physical parameters. The critical value of wave number at which the instability sets increases with the increasing values of $\mu_{1}$. Actually, increasing the values of hot to cold positron concentration ratio is responsible for the decreasing values of the nonlinear coefficient $Q$. As the reason of the values of decreasing nonlinear coefficient the instability sets increases. On the other hand, the absolute value of the ratio $P / Q$ increases with the increasing values of $\mu_{1}$.
We have also analyzed the effect of effective temperature to the hot positron temperature ratio (via $\sigma_{1}$ ) on the stability of the wave profiles [see Fig. 2(b)]. It is observed that the decreasing values of the hot positron temperature (which leads the increasing values of $\sigma_{1}$ ) the instability sets increases. On the other hand, with the decreasing values of the hot electron temperature (which leads the increasing values of $\sigma_{2}$ ) the instability sets decreases [see Fig.2 $(d)$ ]. So increasing hot positron or hot electron temperature plays simultaneously opposite role to recognize the stability region for the PAWs to increase or decrease.

The variation of $P / Q$ with $k$ for different values of hot electron to cold positron concentration ratio (via $\mu_{2}$ ) with fixed values of other physical parameters is depicted in Fig. 
$2(c)$. It is seen that the critical wavenumber decreases with the increasing values of $\mu_{2}$. This leads that the increasing of electron concentration, the critical value $\left(k_{c}\right)$ is shifted to the lower value. So excess number of electron of the system is caused to minimize the stability of the wave profile. Again, the absolute value of the ratio $P / Q$ decreases with the increasing values of $\mu_{2}$.

The variation of MI growth rate (via $\Gamma_{g}$ ) versus MI wave number (via $k_{M I}$ ) is depicted in Fig. $3(a)$. It is observed that the growth rate increases with the increasing values of hot electron to cold positron concentration ratio (via $\mu_{2}$ ). This outcome also implies that the greater (lower) the values of hot electron (cold positron) concentration, the nonlinearity of the PAWs is stimulated (depressed) which expose via the maximum value of MI growth rate. So $\mu_{2}$ plays a vital role to the stability of PAWs profile.

\section{ENVELOPE SOLITONS}

If $P Q<0$, the modulated envelope pulse is stable (in this region, dark envelope solitons exist) and when $P Q>0$, the modulated envelope pulse is unstable against external perturbations and leads to generation of bright envelope solitons. The variation of $P Q$ versus $\mathrm{k}$ is depicted in Fig. 1(a) with different values of nonthermal parameter $\beta$. It is found that with the increasing values of nonthermal parameter $\beta$, the values of critical wave number (when $P Q=0$ ) increases. A solution of (15) may be sought in the form $\Phi=\sqrt{\psi} \exp (i \theta)$, where $\psi$ and $\theta$ are real variables which are determined by substituting into the NLS equation and separating real and imaginary parts. An interested reader is referred to [38]-[42] for details. The different types of solution thus obtained are clearly summarized in the following paragraphs.

\section{A. Bright solitons}

When $P Q>0$, we find bright envelope solitons. The general analytical form of bright solitons reads

$$
\begin{aligned}
& \psi=\psi_{0} \operatorname{sech}^{2}\left(\frac{\xi-U \tau}{W}\right), \\
& \theta=\frac{1}{2 P}\left[U \xi+\left(\Omega_{0}-\frac{U^{2}}{2}\right) \tau\right] .
\end{aligned}
$$

Here, $U$ is the propagation speed (a constant), $W$ is the soliton width, and $\Omega_{0}$ oscillating frequency for $U=0$. Fig. $3(b)$ represents the bright envelope solitons.

\section{B. Dark solitons}

When $P Q<0$, we find dark envelope solitons whose general analytical form reads as

$$
\begin{aligned}
& \psi=\psi_{0} \tanh ^{2}\left(\frac{\xi-U \tau}{W}\right), \\
& \theta=\frac{1}{2 P}\left[U \xi-\left(\frac{U^{2}}{2}-2 P Q \psi_{0}\right) \tau\right] .
\end{aligned}
$$

Interestingly, in both of the latter two equations, the relation between soliton width $W$ and the constant maximum amplitude $\psi_{0}$ are related by

$$
W=\sqrt{\frac{2|P / Q|}{\psi_{0}}} .
$$

Figure 3(c) represents the dark envelope solitons.

\section{CONCLUSION}

We considered an unmagnetized four component e-p-i plasma system consisting of immobile positive ions, inertial mobile cold positrons, and nonthermally distributed hot positrons and hot electrons. The well-known reductive perturbation method has been used to drive a NLS equation, which is valid for a small but finite amplitude limit. We have observed the existence of both stable and unstable regions of PAWs and how the related physical plasma parameters (hot positrons temperature, hot electron temperature, cold electron number density, positron number density and nonthermallity) influence to change the stability conditions of PAWs, MI growth rate, and formation of envelope solitons. Like with the increasing of hot positron (hot electron) concentration, the critical value is shifted to the higher (lower) values of $k$. It is needed to highlights here that the findings of our present investigation should be useful for understanding the striking features of space environments (like cluster explosions, active galactic nuclei, auroral acceleration regions, lower part of magnetosphere, ionosphere etc.) and laboratory plasmas.

\section{ACKNOWLEDGMENT}

N. A. Chowdhury is grateful to the Bangladesh Ministry of Science and Technology for awarding the National Science and Technology (NST) Fellowship.

\section{REFERENCES}

[1] M. C. Begelman, R. D. Blanford, and M. J. Rees, Rev. Mod. Phys. 56, 255 (1992)

[2] H. R. Miller and P. J. Wiita, Active galactic nuclei (Springer, Berlin, 1987).

[3] M. Tribeche, K. Aoutou, S. Younsi, and R. Amour, Phys. Plasmas 16, 072103 (2009)

[4] F. C. Michel, Theory of Neutron Star Magnetosphere (Chicago University Press, Chicago, 1991).

[5] M. R. Hossen and A. A. Mamun, Braz. J. Phys. 44, 673 (2014).

[6] M. R. Hossen, S. A. Ema, and A. A. Mamun, Commun. Theor. Phys. 62, 888 (2014).

[7] W. Misner, K. S. Thorne, and J. I. Wheeler, Gravitation (Freeman, San Francisco, 1973).

[8] M. J. Rees, Nature 229, 312 (1971).

[9] E. P. Liang, S. C. Wilks, and M. Tabak, Phys. Rev. Lett. 81, 4887 (1998).

[10] F. C. Michel, Rev. Mod. Phys. 54, 1 (1982).

[11] M. L. Burns, A. K. Harding, and R. Ramaty, Positron-electron pairs in astrophysics (American Institute of Physics, Melville, New York, 1983).

[12] P. Goldreich and W. H. Julian, Astrophys. J. 157, 869 (1969).

[13] E. Tandberg-Hansen and A. G. Emslie, The Physics of Solar Flares (Cambridge University Press, Cambridge, 1988).

[14] A. S. Bains, A. P. Misra, N. S. Saini, and T. S. Gill, Phys. Plasmas 17, 01203 (2010).

[15] M. A. Rehnam, and M. P. Misra, Phys. Plasmas 23, 012302 (2016).

[16] L. Tie-Lu, W. Yun-Liang, and L. Yan-Zhen, Chin. Phys. B 24, 025202 (2015).

[17] S. Sultana, and I. Kourakis, Plasma Phys.Control. Fusion 53, 045003 (2011).

[18] H. Demiray, Phys. Plasmas 23, 032109 (2016).

[19] Q. Haque, and H. Saleem, Phys. Plasmas 10, 3793 (2003).

[20] A. Shah, R. Saeed, and M. Noaman-Ul-Haq, Phys. Plasmas 17, 072307 (2010).

[21] R. Sabry, W. M. Moslem, and P. K. Sukla, Eur. Phy. J. D 51, 233 (2009).

[22] M. Akbari-Moghshanjoughi, Phys. Plasmas 17, 082315 (2010).

[23] E. I. El-Awady, S. A. El-Tantawy, W. M. Moslem, and P. K. Sukla, Phys. Lett. A 374, 3216 (2010). 
[24] M. S. Alam, M. M. Masud, and A. A. Mamun, Chin. Phys. B 22, 115202 (2013).

[25] H. Matsumoto, H. Kojima, T. Miyatake, I. Nagano, A. Fujita, L. A. Frank, T. Mukai, W. R. Paterson, Y. Saito, S. Machida, and R. R. Anderson, Geophys. Res. Lett. 21, 2915 (1994).

[26] R. Boström, IEEE Trans. Plasma Sci. 20, 756 (1992).

[27] R. Lundin, A. Zakharov, R. Pellinin, H. Borg, B. Hultqvist, N. Pissarenko, E. M. Dubinin, S. W. Barabash, I. Liede, and H. Koskinen, Nature 341, 609 (1989).

[28] P. O. Dovner, A. I. Eriksson, R. Boström, and B. Holback, Geophys. Res. Lett. 21, 1827 (1994)

[29] R. A. Cairns, A. A. Mamun, R. Bingham, R. Boström, R. O. Dendy, C. M. C. Nairn, and P. K. Shukla, Geophys. Res. Lett. 22, 2709 (1995).

[30] P. Chatterjee, D. K. Ghosh, and B. Sahu, Astrophys. Space Sci. 339, 261 (2012).

[31] H. R. PAkzad, Phys. Lett. A 373, 847-850 (2009).

[32] B. Sahu, Phys. Scr. 82,065504 (2010).

[33] A. Messekher, K. Mebrouk, L. A. Gougam, and M. Tribeche, Phys. Plasmas 23, 104504 (2016).

[34] P. Eslami, M. Mottaghizadeh, and H. R. Pakzad, Phys. Plasmas 18, 102313 (2011)

[35] J. Zhang, Y. Wang, and L. Wu, Phys. Plasmas 16, 062102 (2009).

[36] R. Fedele and H. Schamel, Eur. Phys. J. B 27, 313 (2002).

[37] R. Sabry, Phys. Plasmas 15, 092101 (2008).

[38] R. Fedele, Phys. Scr. 65, 502 (2002).

[39] R. Fedele, H. Schamel, and P.K. Sukla, Phys. Scr. T98, 18 (2002).

[40] I. Kourakis and P.K. Sukla, Nonlinear Proc. Geophys. 12, 407 (2005).

[41] Shalini, N. S. Saini, and A. P. Misra, Phys. Plasmas 22, 092124 (2015).

[42] O. Bouzit, M. Tribeche, and A. S. Bains, Phys. Plasmas 22, 084506 (2015). 